\title{
Second harmonic generation in anisotropic magnetic films
}

\author{
V. N. Gridnev, V. V. Pavlov, and R. V. Pisarev \\ A. F. Ioffe Physical Technical Institute of the Russian Academy of Sciences, 194021 St. Petersburg, Russia \\ A. Kirilyuk* and Th. Rasing \\ Research Institute for Materials, University of Nijmegen, Toernooiveld 1, 6525 ED Nijmegen, The Netherlands
}

(Received 18 December 2000; published 17 April 2001)

\begin{abstract}
Nonlinear magneto-optical phenomena related to the process of second harmonic generation in anisotropic films of different classes of symmetry are discussed. In the electric-dipole approximation two kinds of nonlinearity of crystallographic and magnetic origin may coexist in noncentrosymmetric crystals. The interference between the corresponding nonlinear optical waves results in novel magneto-optical effects, such as a transversal effect linear in magnetization at normal incidence and a circular magnetic asymmetry with no equivalence between light helicity and magnetization direction change. Epitaxial films of magnetic garnets grown on substrates with different crystallographic orientations were taken as a model anisotropic system for experimental studies. The unambiguous separation of the crystallographic and magnetic contributions to the second harmonic generation is demonstrated with the help of rotational anisotropy experiments. A theoretical model for the nonlinear light propagation in noncentrosymmetric magnetic films is developed. Calculations based on this model are found to be in good agreement with the experimental results.
\end{abstract}

DOI: 10.1103/PhysRevB.63.184407

PACS number(s): 75.50.Gg, 78.20.Ls, 42.65.Ky, 75.70.-i

\section{INTRODUCTION}

The field of nonlinear optics started in 1961 by the second harmonic generation (SHG) experiment of Franken et al. ${ }^{1}$ Numerous nonlinear optical phenomena have been discovered since then and several comprehensive textbooks were written on this subject (e.g., Refs. 2-5). Within the vast area of nonlinear optics, second harmonic generation plays a very essential role. Being a higher-order process, it brings new and complementary information in comparison with linear optics, partly due to the different selection rules for multiphoton processes and the higher-order susceptibilities involved. Another strong point of the technique is its intrinsic surface and interface sensitivity that is derived from extremely simple yet powerful symmetry constraints. The SHG technique was therefore widely used for studies of surfaces and interfaces. ${ }^{5-7}$

The breaking of time-reversal symmetry leads to a number of well known magneto-optical effects such as Faraday rotation in transmission and Kerr rotation in reflection. ${ }^{8}$ For nonlinear optics, in the electric dipole approximation, evenorder effects such as SHG are only allowed in media with a broken space inversion symmetry. As a consequence, nonlinear magneto-optical effects can only be observed in materials in which both space-inversion and time reversal symmetry are broken. Thought the first predictions of magnetic effects in SHG were made over 30 years ago ${ }^{9,10}$ and discussed in several theoretical publications, ${ }^{11-14}$ the field of nonlinear magneto-optics really evolved in the past decade by observation of huge magneto-optical effects from magnetic surfaces and interfaces. ${ }^{15-17}$ These strong magneto-optical effects have already been successfully applied for the study of magnetic thin films and multilayers, ${ }^{18-20}$ that are important for applications in future so-called spin-electronic devices. In addition, it has been demonstrated that, conform the original predictions, SHG can be used to study the antiferromagnetic ordering and even image antiferromagnetic domains which is very hard or even impossible to do with other techniques. ${ }^{21,22}$

This paper presents a theoretical and experimental study of nonlinear magneto-optical phenomena in anisotropic magnetic films. The origin of these phenomena lies in the two sources of nonlinear polarization which may coexist in noncentrosymmetric magnetic crystals. The crystallographic contribution arises in the electric-dipole approximation due to the space-inversion symmetry breaking whereas the magnetic contribution arises due to the time-reversal symmetry breaking. The interference between the nonlinear optical waves coming from these two sources results in new nonlinear optical phenomena, that do not exist in linear magnetooptics, such as transversal nonlinear magneto-optical effects linear in the magnetization and the breaking of the equivalence between magnetization reversal and the change in the light helicity. In some cases, for high symmetry directions, an SHG signal is generated only in the presence of a magnetization.

Magnetic garnet films are a well known group of materials characterized by a large variety of magnetic, optical, and magneto-optical properties. ${ }^{23-26}$ Several experiments showed that SHG can be readily observed in these films, ${ }^{27-29}$ indicating that for garnet films the inversion symmetry is broken in the originally centrosymmetric bulk garnet structure. The magnetic contribution of the SHG was proven to exist only recently. ${ }^{30,31}$ In modeling the nonlinear light propagation in magnetic films we will show that the linear magneto-optical effects must be taken into account in the longitudinal geometry but they are not essential in the transversal one. Analyzing the symmetry properties of the two contributions to SHG from magnetic films of different symmetries we show how they can be unambiguously separated.

The paper is organized as follows. Section II describes the theoretical model of nonlinear magneto-optics in magnetic films, giving a general description of optical tensors (Sec. 
II A) and the nonlinear propagation of light in magnetic films (Sec. II B). Section II C deals with the transformation symmetry properties of different contributions to SHG and the calculation of the total SHG intensity (Sec. IID). After a brief consideration of the experimental details (Sec. III) we present the experimental results and a discussion of different nonlinear magneto-optical phenomena in Sec. IV, followed by a Conclusion (Sec. V).

\section{THEORY}

\section{A. General description of optical tensors}

An incident light wave induces a polarization in a medium that serves as a source for the transmitted and reflected light. The polarization $\mathbf{P}$ can be written in the electric-dipole approximation as an expansion in powers of the optical electric field $\mathbf{E}(\omega)$ :

$$
\mathbf{P}(\omega, 2 \omega, \ldots)=\hat{\chi}^{l} \mathbf{E}(\omega)+\hat{\chi}^{n l} \mathbf{E}(\omega) \mathbf{E}(\omega)+\cdots .
$$

The tensor $\hat{\chi}^{l}$ is the linear optical susceptibility allowed in all media. SHG is described by the second term with the corresponding nonlinear tensor $\hat{\chi}^{n l}$ allowed only in noncentrosymmetric media. For crystals with a spontaneous or magnetic-field induced magnetization $\mathbf{M}$, the nonlinear optical polarization of a medium $\mathbf{P}^{n l}(2 \omega)$ can be written as

$$
\mathbf{P}^{n l}(2 \omega)=\hat{\chi}^{c r} \mathbf{E}(\omega) \mathbf{E}(\omega)+\hat{\chi}^{m} \mathbf{E}(\omega) \mathbf{E}(\omega) \mathbf{M},
$$

where the first term describes the purely crystallographic contribution while the second one only exists in the presence of a magnetization $\mathbf{M}$ and describes magnetization-induced SHG. The two contributions to the nonlinear polarization $\mathbf{P}^{n l}(2 \omega)$ are of electric-dipole character and simultaneously allowed in noncentrosymmetric media, but their properties are different. The crystallographic contribution is described by a polar tensor $\hat{\chi}^{c r}$ of rank 3 , whereas the magnetizationinduced contribution is described by an axial tensor $\hat{\chi}^{m}$ of rank 4. In lossless media $\hat{\chi}^{c r}$ is a real tensor and $\hat{\chi}^{m}$ is an imaginary tensor, therefore there is no interference between the SHG waves coming from these two sources for linear polarized fundamental light. ${ }^{9,13}$ The interference becomes allowed for linear polarized fundamental light in the absorption region, because both tensors will be complex, or by using circular optical excitation. This interference gives rise to new nonlinear magneto-optical effects which have no counterparts in linear optics.

\section{B. Nonlinear propagation of light in magnetic films}

The calculation of the SHG intensity using Eq. (2) requires knowledge of $\mathbf{E}(\omega)$. Therefore the linear optical effects coming from the light propagation in a magnetic medium and described by $\hat{\chi}^{l}$ should be taken into account simultaneously with the nonlinear optical effects described by $\hat{\chi}^{n l}$. This is especially important for the experiments in transmission, where linear optical effects can be large.

Consider an SHG electric field $\mathbf{E}(\mathbf{r}, 2 \omega)$ induced by the fundamental electric field $\mathbf{E}(\mathbf{r}, \omega)=\mathbf{A} e^{i(\mathbf{k r}-\omega t)}$ incident from

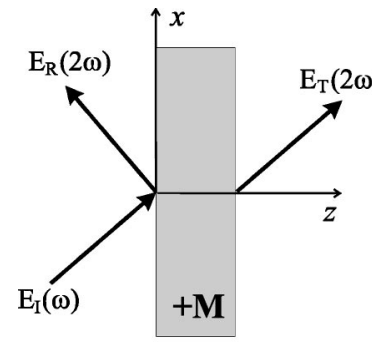

(a)

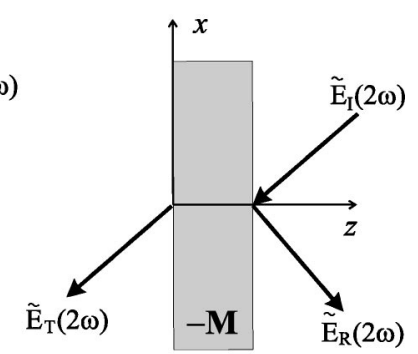

(b)
FIG. 1. Geometry of SHG from a magnetized film: (a) original nonlinear problem, (b) illustration of the origin of the auxiliary solution $\widetilde{\mathbf{E}}(2 \omega)$ of the linear problem.

vacuum on a crystalline magnetized film of thickness $d$ at an angle $\theta$, Fig. 1(a). The film occupies the space $0<z<d$. The field $\mathbf{E}(\mathbf{r}, 2 \omega)$ obeys the wave equation

$$
\begin{aligned}
\boldsymbol{\nabla} & \times \boldsymbol{\nabla} \times \mathbf{E}(\mathbf{r}, 2 \omega)-\frac{(2 \omega)^{2}}{c^{2}} \varepsilon(2 \omega) \mathbf{E}(\mathbf{r}, 2 \omega) \\
& =4 \pi \frac{(2 \omega)^{2}}{c^{2}} \mathbf{P}(\mathbf{r}, 2 \omega)
\end{aligned}
$$

The nonlinear polarization, induced inside the film, is given by

$$
\mathbf{P}(\mathbf{r}, 2 \omega)=\int \hat{\chi}^{n l}\left(\mathbf{r}, \mathbf{r}^{\prime}, \mathbf{r}^{\prime \prime}, 2 \omega\right) \mathbf{E}\left(\mathbf{r}^{\prime}, \omega\right) \mathbf{E}\left(\mathbf{r}^{\prime \prime}, \omega\right) d \mathbf{r}^{\prime} d \mathbf{r}^{\prime \prime}
$$

The coordinate dependence of the second-order nonlinear susceptibility $\hat{\chi}^{n l}\left(\mathbf{r}, \mathbf{r}^{\prime}, \mathbf{r}^{\prime \prime}, 2 \omega\right)$ takes into account the presence of boundaries as well as effects of spatial dispersion. The latter are important only in a medium which possesses a center of inversion and they become small in comparison with the electric-dipole effects in a noncentrosymmetric medium. Here we discuss the transmitted SHG wave $\mathbf{E}_{T}(\mathbf{r}, 2 \omega)=\mathbf{T} e^{2 i(\mathbf{k r}-\omega t)}$, where $\mathbf{T}$ represents the complex amplitude of the wave. The amplitude $\mathbf{T}$ may be calculated by using Eq. (6) of Ref. 32:

$$
\cos \theta(\widetilde{\mathbf{A}} \mathbf{T})=i \frac{4 \pi \omega}{c} \int_{0}^{d} \widetilde{\mathbf{E}}(\mathbf{r}, 2 \omega) \mathbf{P}(\mathbf{r}, 2 \omega) d z,
$$

where $\widetilde{\mathbf{E}}(\mathbf{r}, 2 \omega)$ is an auxiliary solution of the linear transmission problem in which the light wave $\widetilde{\mathbf{E}}_{I}(\mathbf{r}, 2 \omega)$ $=\widetilde{\mathbf{A}} e^{2 i(-\mathbf{k r}-\omega t)}$ is incident on the film along the direction of the reversed wave vector $\mathbf{- k}$ from the opposite side of the film, as is shown in Fig. 1(b). Obviously, the wave $\widetilde{\mathbf{E}}_{I}(\mathbf{r}, 2 \omega)$ propagates in the opposite direction to the SHG transmitted wave $\mathbf{E}_{T}(\mathbf{r}, 2 \omega)$. Another feature of this auxiliary solution is that the magnetization of the film is equal to $-\mathbf{M}$, i.e., the sign of the magnetization is reversed (see Ref. 32 for details).

In order to calculate $\mathbf{T}$ by using Eq. (5) the auxiliary solution $\widetilde{\mathbf{E}}(\mathbf{r}, 2 \omega)$ of the linear transmission problem and nonlinear polarization $\mathbf{P}(\mathbf{r}, 2 \omega)$ should be found. Whereas the 
calculation of $\widetilde{\mathbf{E}}(\mathbf{r}, 2 \omega)$ does not encounter any serious difficulties, the evaluation of $\mathbf{P}(\mathbf{r}, 2 \omega)$ requires the solution of the nonlinear problem. Fortunately, it is possible in this case to take a commonly accepted approximation and calculate the nonlinear polarization $\mathbf{P}(\mathbf{r}, 2 \omega)$ using lowest-order perturbation theory, i.e., one may neglect nonlinear effects solving the linear transmission problem for the fundamental field $\mathbf{E}(\mathbf{r}, \omega)$ and then use $\mathbf{E}(\mathbf{r}, \omega)$ as the fundamental field that creates $\mathbf{P}(\mathbf{r}, 2 \omega)$. Then Eq. (5) gives the explicit representation for the transmitted SHG field through two solutions of the linear transmission problem. To obtain, e.g., the $S$ component of the SHG transmitted field, one should find the solutions for the two linear transmission problems. First, the fundamental field $\mathbf{E}(\mathbf{r}, \omega)$ and the nonlinear polarization source $\mathbf{P}(\mathbf{r}, 2 \omega)$ should be found. Second, one should take the $S$-polarized wave $\widetilde{\mathbf{E}}_{I}(\mathbf{r}, 2 \omega)$, incoming along $-\mathbf{k}$, reverse the magnetization of the film and find the auxiliary solution $\widetilde{\mathbf{E}}(\mathbf{r}, 2 \omega)$ of the transmission problem. Then, the integration in Eq. (5) gives the $S$ component of the reflected SHG field. We emphasize that Eq. (5) takes into account nonlinear optical effects described by $\hat{\chi}^{n l}$ simultaneously with the linear effects of light propagation in a magnetic medium. This is especially important for the experiments in transmission, where the amplitudes of fundamental and SHG light may strongly vary due to the Faraday effect.

Following this method we calculate the SHG transmitted wave in the case of normal incidence. We neglect the linear birefringence of the film, but take into account the Faraday effect which occurs in the longitudinal geometry $(\mathbf{M} \| \mathbf{k})$. The solutions of the linear problems are quite obvious in this case. The fundamental field inside the film has the form

$$
\mathbf{E}(z, \omega) \propto \mathbf{a}^{+} e^{i k^{+} z}+\mathbf{a}^{-} e^{i k^{-} z},
$$

where amplitudes $\mathbf{a}^{ \pm} \propto\left(A_{x} \mp i A_{y}\right)\left(\mathbf{e}_{x} \pm i \mathbf{e}_{y}\right)$ are defined through the amplitude $\mathbf{A}$ of the incident light $k^{ \pm}$ $=(\omega / c) \sqrt{\varepsilon_{0}(\omega) \pm i \varepsilon_{x y}(\omega, \mathbf{M})}$ are the wave vectors of the right and left circularly polarized components of the propagating light. The off-diagonal component of the optical dielectric tensor $\varepsilon_{x y}$ is a linear function of magnetization $\mathbf{M}$ and describes the Faraday effect. ${ }^{8}$ For the transversal magnetization $(\mathbf{M} \perp \mathbf{k}) \varepsilon_{x y}=0$.

The auxiliary solution of the linear problem required for the calculation of the SHG electric field by using Eq. (5) has a similar form

$$
\widetilde{\mathbf{E}}(2 \omega, z) \propto \mathbf{b}^{+} e^{i k_{2}^{-}(d-z)}+\mathbf{b}^{-} e^{i k_{2}^{+}(d-z)},
$$

where quantities $\mathbf{b}^{ \pm}$and $k_{2}^{ \pm}=k_{2}^{ \pm}(\mathbf{M})$ are defined similar to those in Eq. (6) and we use the relation $k_{2}^{ \pm}(\mathbf{M})=k_{2}^{\mp}(-\mathbf{M})$. This solution corresponds to the reversed magnetization of the film and the eigenmodes $\mathbf{e}^{ \pm}=\left(\mathbf{e}_{x} \pm i \mathbf{e}_{y}\right)$ propagate now with wave vectors $k_{2}^{\mp}$, respectively. As is seen from Eq. (5) for calculating $T_{i}$ with $i=x$ or $y$ one should take $\tilde{\mathbf{A}}$ polarized along $x$ or $y$, respectively.

Using Eq. (5) for the calculation of $\mathbf{T}$ we assume that $\hat{\chi}^{n l}\left(\mathbf{r}, \mathbf{r}^{\prime}, \mathbf{r}^{\prime \prime}, 2 \omega\right)$ is independent of the coordinates inside the film. The substitution of Eqs. (4), (6), and (7) into Eq. (5)
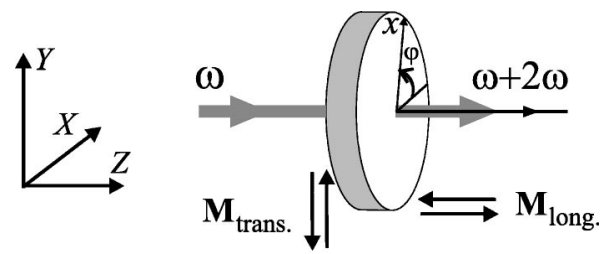

FIG. 2. Experimental geometry. The sample frame is $x y z$ and the laboratory frame is $X Y Z$. Sample can be rotated while the magnetization is fixed in the laboratory frame.

and the integration over $z$ give the following expression for the amplitude of the outgoing SHG field:

$$
T_{x, y} \propto \sum_{\substack{i, k, l \\ q, u, v= \pm}} \chi_{i k l} a_{k}^{q} a_{l}^{u} b_{i}^{-v} \frac{e^{i d k_{2}^{v}-e^{i d\left(k^{q}+k^{u}\right)}}}{k^{q}+k^{u}-k_{2}^{v}} .
$$

The dependence of this equation on the corresponding component of the transmitted SHG wave ( $x$ or $y$ ) is implicitly contained in the amplitudes $b_{i}^{ \pm}$.

Equation (8) will be used below for fitting the experimental SHG data in the magnetic films under study. For the transversal geometry Eq. (8) is greatly simplified because the wave vectors $k^{u}$ and $k_{2}^{u}$ do not depend on $u= \pm$. In this case we obtain a simple result $T_{i} \propto P_{i}(2 \omega)=\chi_{i j k} E_{j}(\omega) E_{k}(\omega)$. However, this is not the case if SHG is studied in the longitudinal magneto-optical geometry. The Faraday effect strongly affects the propagating light at both $\omega$ and $2 \omega$ frequencies in this case. For this reason one should perform a numerical summation on $q, u$ and $v$ in Eq. (8) in order to give the quantitative description of nonlinear magnetooptical effects.

\section{Symmetry considerations}

The symmetry properties of the tensors $\hat{\chi}^{c r}$ and $\hat{\chi}^{m}$ are strictly defined by the crystallographic point group (PG). All relevant nonlinear magneto-optical effects defined by the tensors $\hat{\chi}^{c r}$ and $\hat{\chi}^{m}$ in addition must depend on the mutual directions of polarization of light, crystal axes, and the magnetization orientation.

A suitable way to study the transformation properties of the crystallographic and magnetization-induced contributions to SHG is the rotational anisotropy method, i.e., to measure the SHG intensity as a function of the sample azimuthal angle $\varphi$ (see Fig. 2). Below we derive the expressions describing the rotational anisotropy patterns for the crystallographic and magnetization-induced contributions to SHG in anisotropic crystals of several symmetry classes.

\section{Crystallographic contribution to SHG}

Figure 2 shows the geometry considered. The sample $x y z$ frame rotates with respect to the laboratory frame $X Y Z$ in such a way that $z \| Z$, with both fundamental and SHG light propagating along the sample normal $\mathbf{k}\left\|\mathbf{k}_{2}\right\| \mathbf{n} \| z$. In order to calculate the outgoing SHG intensity behind the analyzer, one needs to know the induced nonlinear polarization $\mathbf{P}(2 \omega)$ in the laboratory frame. The nonlinear polarization induced 
TABLE I. Relevant components of the nonlinear optical tensors $\hat{\chi}^{c r}$ and $\hat{\chi}^{m}$ for the geometry shown in Fig. 2 .

\begin{tabular}{lcc}
\hline \hline$\chi_{i j k}^{c r}$ & $\chi_{i j k l}^{m}(\mathbf{M} \| Y)$ & $\chi_{i j k l}^{m}(\mathbf{M} \| Z)$ \\
\hline$x x x=A$ & $x x x x=a$ & $x x x z=l$ \\
$y y y=B$ & $y y y y=b$ & $y y y z=m$ \\
$x x y=x y x=C$ & $x x x y=c$ & $x x y z=x y x z=n$ \\
$y x x=D$ & $x y x x=x x y x=d$ & $y x x z=p$ \\
$y y x=y x y=F$ & $y x x x=d_{1}$ & $y y x z=y x y z=s$ \\
$x y y=G$ & $y y y x=f$ & $x y y z=t$ \\
\multicolumn{4}{c}{} \\
$y x y y=y y x y=g$ \\
$x y y y=g_{1}$ \\
$x x y=x y x y=h$ \\
$x y y x=h_{1}$ \\
$y x x=y x y x=k$ \\
$y x x y=k_{1}$ \\
\hline
\end{tabular}

in the sample is given by $p_{i}=\chi_{i j k}^{c r} e_{j} e_{k}$, where $p_{i}, e_{j}$, and $e_{k}$ are the nonlinear polarization and fundamental electric field components in the sample frame, respectively. It is straightforward to write the transformation formulas for the fields

$$
\begin{gathered}
e_{x}=E_{X} \cos \varphi+E_{Y} \sin \varphi, \\
e_{y}=-E_{X} \sin \varphi+E_{Y} \cos \varphi,
\end{gathered}
$$

and the polarizations

$$
\begin{aligned}
& P_{X}=p_{x} \cos \varphi-p_{y} \sin \varphi, \\
& P_{Y}=p_{x} \sin \varphi+p_{y} \cos \varphi .
\end{aligned}
$$

Table I shows the components of the nonlinear optical tensors $\hat{\chi}^{c r}$ and $\hat{\chi}^{m}$ that are relevant in the geometry of Fig. 2. Using the notations of this table and Eqs. (9) and (10) one can derive for the nonlinear polarization $P_{I J}^{c r}$ the following expressions:

$$
\begin{aligned}
P_{X X}^{c r}= & E_{X}^{2}\left[A \cos ^{3} \varphi-(D+2 C) \cos ^{2} \varphi \sin \varphi\right. \\
& \left.+(G+2 F) \cos \varphi \sin ^{2} \varphi-B \sin ^{3} \varphi\right], \\
P_{X Y}^{c r}= & E_{X}^{2}\left[D \cos ^{3} \varphi+(A-2 F) \cos ^{2} \varphi \sin \varphi\right. \\
& \left.+(B-2 C) \cos \varphi \sin ^{2} \varphi+G \sin ^{3} \varphi\right], \\
P_{Y X}^{c r}= & E_{Y}^{2}\left[G \cos ^{3} \varphi-(B-2 C) \cos ^{2} \varphi \sin \varphi\right. \\
& \left.+(A-2 F) \cos \varphi \sin ^{2} \varphi-D \sin ^{3} \varphi\right], \\
P_{Y Y}^{c r}= & E_{Y}^{2}\left[B \cos ^{3} \varphi+(G+2 F) \cos ^{2} \varphi \sin \varphi\right. \\
& \left.+(D+2 C) \cos \varphi \sin ^{2} \varphi+A \sin ^{3} \varphi\right] .
\end{aligned}
$$

\begin{tabular}{|c|c|c|c|c|}
\hline PG & $\hat{\chi}^{c r}$ & $\hat{\chi}^{m}(\mathbf{M} \| Y)$ & $\hat{\chi}^{m}(\mathbf{M} \| Z)$ & $h k l$ \\
\hline 1 & $\begin{array}{c}A, B, C, \\
D, F, G\end{array}$ & $\begin{array}{c}a, b, c, d, \\
d_{1}, f, g, g_{1}, \\
h, h_{1}, k, k_{1}\end{array}$ & $\begin{array}{c}l, m, n, \\
p, s, t\end{array}$ & (110) \\
\hline $\begin{array}{l}m \\
(z \|[210])\end{array}$ & $B, C, D$ & $\begin{array}{l}c, d, d_{1} \\
f, g, g_{1}\end{array}$ & $l, s, t$ & (210) \\
\hline $3 m$ & $\begin{array}{c}A=-F \\
=-G\end{array}$ & $\begin{array}{c}c=-2 d-d_{1} \\
=-f=2 g+g_{1}\end{array}$ & $\begin{array}{c}m=-n \\
=-p\end{array}$ & (111) \\
\hline$m m 2$ & & $c, d, d_{1}, f, g, g_{1}$ & & (110) \\
\hline $4 \mathrm{~mm}$ & & $\begin{array}{c}c=-f, d=-g \\
d_{1}=-g_{1}\end{array}$ & & (001) \\
\hline
\end{tabular}

For $P_{I J}^{c r}$ the subscripts $I$ and $J$ denote the fundamental and SHG linear polarizations, respectively.

Crystal symmetry imposes restrictions on the components of the nonlinear tensors $\hat{\chi}^{c r}$ and $\hat{\chi}^{m}$. Table II shows the nonvanishing components of the nonlinear tensors for several PGs discussed in this paper. The crystallographic contribu-
TABLE II. Nonzero components of the $\hat{\chi}^{c r}$ and $\hat{\chi}^{m}$ tensors for several PG relevant in the transversal $(\mathbf{M} \| Y)$ and longitudinal geometries $(\mathbf{M} \| Z)$.

tion to the nonlinear polarization $\mathbf{P}(2 \omega)$ vanishes in the considered geometry for the PG $4 \mathrm{~mm}$ and $\mathrm{mm} 2$.

In addition to Eqs. (11), one can derive similar expressions for circularly polarized incident light. For this purpose, Eqs. (9) should be substituted by

$$
e_{x}=E_{0} e^{ \pm i \varphi}, \quad e_{y}= \pm i E_{0} e^{ \pm i \varphi},
$$

where \pm signs refer to the right/left helicity of the incoming light, respectively. Thus for the nonlinear polarizations $P_{C J}^{c r}$ (where $C$ denotes the circular polarization of the incoming light) one gets

$$
\begin{aligned}
P_{C X}^{c r}= & E_{0}^{2} e^{ \pm 2 i \varphi}[(A-G) \cos \varphi \pm 2 i(C \cos \varphi-F \sin \varphi) \\
& +(B-D) \sin \varphi], \\
P_{C Y}^{c r}= & E_{0}^{2} e^{ \pm 2 i \varphi}[(D-B) \cos \varphi \pm 2 i(F \cos \varphi+C \sin \varphi) \\
& +(A-G) \sin \varphi] .
\end{aligned}
$$

\section{Magnetization-induced contribution to SHG in the transversal geometry}

In order to derive the nonlinear polarization $P_{I J}^{m t}$ induced by the sample magnetization $\mathbf{M}$ in the transversal geometry with $\mathbf{M} \| Y$ (see Fig. 2) we use the second column of Table I. The transformation formulas for the magnetization $\mathbf{M}$ can be written as

$$
m_{x}=M_{Y} \sin \varphi, \quad m_{y}=M_{Y} \cos \varphi .
$$

The nonlinear polarization in the sample frame is given by $p_{i}=\chi_{i j k l}^{m} e_{j} e_{k} m_{l}$. Together with Eqs. (9), (10), and (14) this results in the magnetization-induced nonlinear polarization for the transversal geometry

$$
\begin{aligned}
P_{X X}^{m t}= & E_{X}^{2} M_{Y}\left[c \cos ^{4} \varphi+\left(a-k_{1}-2 h\right) \cos ^{3} \varphi \sin \varphi\right. \\
& +\left(2 g+g_{1}-2 d-d_{1}\right) \cos ^{2} \varphi \sin ^{2} \varphi \\
& \left.+\left(h_{1}-b+2 k\right) \cos \varphi \sin ^{3} \varphi-f \sin ^{4} \varphi\right],
\end{aligned}
$$




$$
\begin{aligned}
P_{X Y}^{m t}= & E_{X}^{2} M_{Y}\left[k_{1} \cos ^{4} \varphi+\left(c+d_{1}-2 g\right) \cos ^{3} \varphi \sin \varphi\right. \\
& +(a+b-2 k-2 h) \cos ^{2} \varphi \sin ^{2} \varphi \\
& \left.+\left(g_{1}+f-2 d\right) \cos \varphi \sin ^{3} \varphi+h_{1} \sin ^{4} \varphi\right], \\
P_{Y X}^{m t}= & E_{Y}^{2} M_{Y}\left[g_{1} \cos ^{4} \varphi+\left(2 h+h_{1}-b\right) \cos ^{3} \varphi \sin \varphi\right. \\
& +(c+2 d-f-2 g) \cos ^{2} \varphi \sin ^{2} \varphi \\
& \left.+\left(a-2 k-k_{1}\right) \cos \varphi \sin ^{3} \varphi-d_{1} \sin ^{4} \varphi\right], \\
P_{Y Y}^{m t}= & E_{Y}^{2} M_{Y}\left[b \cos ^{4} \varphi+\left(f+2 g+g_{1}\right) \cos ^{3} \varphi \sin \varphi\right. \\
& +\left(2 k+k_{1}+2 h+h_{1}\right) \cos ^{2} \varphi \sin ^{2} \varphi \\
& \left.+\left(c+2 d+d_{1}\right) \cos \varphi \sin ^{3} \varphi+a \sin ^{4} \varphi\right] .
\end{aligned}
$$

The nonzero components of the nonlinear tensors are shown in Table II for the considered geometry.

\section{Magnetization-induced contribution to SHG in the longitudinal geometry}

The magnetization-induced nonlinear polarization for the longitudinal geometry with $\mathbf{M} \| Z$ can be written as

$$
\begin{aligned}
P_{X X}^{m l}= & E_{X}^{2} M_{z}\left[l \cos ^{3} \varphi-(p+2 n) \cos ^{2} \varphi \sin \varphi\right. \\
& \left.+(t+2 s) \cos \varphi \sin ^{2} \varphi-m \sin ^{3} \varphi\right], \\
P_{X Y}^{m l}= & E_{X}^{2} M_{z}\left[p \cos ^{3} \varphi+(l-2 s) \cos ^{2} \varphi \sin \varphi\right. \\
& \left.+(m-2 n) \cos \varphi \sin ^{2} \varphi+t \sin ^{3} \varphi\right], \\
P_{Y X}^{m l}= & E_{Y}^{2} M_{z}\left[t \cos ^{3} \varphi-(m-2 n) \cos ^{2} \varphi \sin \varphi\right. \\
& \left.+(l-2 s) \cos \varphi \sin ^{2} \varphi-p \sin ^{3} \varphi\right], \\
P_{Y Y}^{m l}= & E_{Y}^{2} M_{z}\left[m \cos { }^{3} \varphi+(t+2 s) \cos ^{2} \varphi \sin \varphi\right. \\
& \left.+(p+2 n) \cos \varphi \sin ^{2} \varphi+l \sin ^{3} \varphi\right] .
\end{aligned}
$$

The corresponding tensor components are shown in Tables I and II. Similarly to the crystallographic contribution, the magnetization-induced nonlinear polarization in the longitudinal geometry vanishes for the PG $4 \mathrm{~mm}$ and $\mathrm{mm} 2$.

In the longitudinal geometry, using circular polarization for the incoming light [Eqs. (12)] one gets the following expressions for the magnetization-induced nonlinear polarizations:

$$
\begin{aligned}
P_{C X}^{m l}= & E_{0}^{2} M_{z} e^{ \pm 2 i \varphi}[(l-t) \cos \varphi \pm 2 i(n \cos \varphi-s \sin \varphi) \\
& +(m-p) \sin \varphi] \\
P_{C Y}^{m l}= & E_{0}^{2} M_{z} e^{ \pm 2 i \varphi}[(p-m) \cos \varphi \pm 2 i(s \cos \varphi+n \sin \varphi) \\
& +(l-t) \sin \varphi] .
\end{aligned}
$$

\section{Calculation of SHG intensity}

As was pointed out in Sec. II B, the linear magnetooptical Faraday effect vanishes in the transversal geometry and the resulting SHG intensity $I(2 \omega)$ can be written as

$$
I(2 \omega) \propto\left|P_{I J}^{c r}+P_{I J}^{m}\right|^{2},
$$

where both $P_{I J}^{c r}$ and $P_{I J}^{m}$ are taken for the corresponding fundamental and SHG light polarization combinations. In absorbing media the complex character of the nonlinear optical tensor components should be always explicitly taken into account, because in this case the interference between the crystallographic and magnetization-induced parts of the nonlinear wave is allowed. This gives rise to magnetization induced changes of the SHG intensity. Equation (18) is still valid for the longitudinal geometry in the case of a weak Faraday effect or when the thickness of the magnetic film is much smaller than the wavelength of light. In other cases for the longitudinal geometry one should solve the problem of the propagation of the SHG light and use Eq. (8) for calculating the resulting SHG intensity $I(2 \omega)$.

\section{EXPERIMENTAL DETAILS}

\section{A. Samples}

Crystallographic, magnetic, optical, and magneto-optical properties of bulk crystals and thin films of magnetic garnets are thoroughly discussed in several books and review papers, see, e.g., Refs. 23-26. Bulk crystals of magnetic garnets such as the well known yttrium iron garnet $\mathrm{Y}_{3} \mathrm{Fe}_{5} \mathrm{O}_{12}$, belong to the cubic centrosymmetric PG $m 3 m$ (space group $I a 3 d)$. Consequently, SHG in garnets is forbidden in the electric-dipole approximation and we are unaware of any experimental reports concerning the observation of SHG in bulk garnet crystals. The garnet unit cell contains eight formula units. The ferrimagnetic structure of $\mathrm{Y}_{3} \mathrm{Fe}_{5} \mathrm{O}_{12}$ is formed by two oppositely oriented octahedral and tetrahedral, magnetic sublattices, with cubic magnetic anisotropy and the magnetization orientation along the [111] axes. This type of magnetic ordering does not destroy the spaceinversion symmetry of the magnetic unit cell and thus cannot induce a bulk SHG of electric-dipole type. To check this experimentally we studied SHG in thin platelets cut from bulk crystals of $\mathrm{Y}_{3} \mathrm{Fe}_{5-x} \mathrm{Ga}_{x} \mathrm{O}_{12}(x \approx 0.7)$. The SHG signals in these samples were several orders of magnitude lower than the SHG signals from the thin garnet films and could be related to surface electric-dipole or bulk quadrupole contributions.

Epitaxial films of magnetic garnets used in the present study were grown by a liquid phase epitaxial method. Films were grown on substrates cut from bulk cubic crystals of gadolinium gallium garnet $\mathrm{Gd}_{3} \mathrm{Ga}_{5} \mathrm{O}_{12}$ (GGG) or substituted GGG with a different lattice parameter and with orientations (001), (110), (111), and (210). Samples differed in their compositions and lattice parameters. In total, more than 20 samples were studied. Some important parameters of the most investigated samples are given in Table III. SHG was detected in all of them, though the intensity of the signal could vary by one or two orders of magnitude. The strongest SHG signals were detected in (210) films.

Thin garnet films, in contrast to bulk crystals, are characterized by a noncubic magnetic anisotropy. Several models were proposed to explain the origin of the growth-induced noncubic anisotropy that basically is related to the ordering 
TABLE III. Chemical composition and crystallographic parameters of magnetic garnet films used for the present study. $a_{\text {substrate }}$ and $a_{\text {film }}$ are the lattice parameters of substrate and film, respectively.

\begin{tabular}{lccccc}
\hline \hline No. & Composition & $h k l$ & $a_{\text {substrate }}(\mathrm{nm})$ & $a_{\text {film }}(\mathrm{nm})$ & Misfit $(\%)$ \\
\hline 1 & $(\mathrm{YBiPrLu})_{3}(\mathrm{FeGa})_{5} \mathrm{O}_{12}$ & $(210)$ & $1.24899(12)$ & $1.25047(11)$ & 0.06 \\
2 & $(\mathrm{YBiPrLu})_{3}(\mathrm{FeGa})_{5} \mathrm{O}_{12}$ & $(210)$ & $1.24789(8)$ & $1.25276(8)$ & 0.19 \\
3 & $(\mathrm{YBiLu})_{3}(\mathrm{FeGa})_{5} \mathrm{O}_{12}$ & $(111)$ & $1.23822(12)$ & $1.24138(10)$ & 0.13 \\
4 & $\left(\mathrm{Eu}_{2.5} \mathrm{Lu}_{0.5}\right)\left(\mathrm{Fe}_{4.3} \mathrm{Al}_{0.7}\right) \mathrm{O}_{12}$ & $(111)$ & & $1.2495(1)$ & \\
5 & $(\mathrm{YBi})_{3}(\mathrm{FeGa})_{5} \mathrm{O}_{12}$ & $(110)$ & $1.23796(10)$ & $1.2388(2)$ & 0.03 \\
6 & $(\mathrm{YEuLuCa})_{3}(\mathrm{FeGe})_{5} \mathrm{O}_{12}$ & $(110)$ & $1.2379(2)$ & $1.243(1)$ & 0.2 \\
7 & $(\mathrm{YPr})_{3}(\mathrm{FeGa})_{5} \mathrm{O}_{12}$ & $(100)$ & $1.23787(11)$ & $1.24140(15)$ & 0.14 \\
\hline \hline
\end{tabular}

of ions on the nonequivalent crystallographic sites in the unit cell during the growth process of the films and to the nonuniform deformation of the garnet films due to the lattice mismatch. The deviations of the crystal symmetry from cubic are easily detectable by magnetic or optical birefringence measurements. Moreover, the observation of the linear magneto-electric effect in thin magnetic garnet films ${ }^{33}$ proves that, in addition to the noncubic distortion of the crystal structure, the space-inversion symmetry is broken as well. This point is not important in the analysis of the magnetic properties of the films, but it plays an essential role for the linear and especially for the nonlinear optical properties. For example, it allows the crystallographic as well as the magnetic SHG in the electric-dipole approximation. ${ }^{30,31}$

\section{B. Experimental technique}

Plane-parallel samples were placed on a rotatable sample holder attached to a stepping motor. Experiments were done in transmission at normal incidence with a laser beam propagating along the $Z$ axis (see Fig. 2). Rotating the sample around the $Z$ axis the SHG signals could be registered as a function of the azimuthal angle $\varphi$ in the range $0 \leqslant \varphi \leqslant 360^{\circ}$. A magnetic field up to $H=3 \mathrm{kOe}$ was applied either along the $Z$ axis (longitudinal geometry) or along the $Y$ axis (transversal geometry). The saturation of the magnetization in the applied field was checked for all samples by measurements of the field dependences of the Faraday effect at the fundamental frequency. Such an approach allows unambiguous separation between crystallographic and magnetizationinduced SHG signals. In the absence of a magnetic field these measurements provide the rotational anisotropy of the SHG signals due to the crystallographic contributions, but they do not allow the magnetic field dependent part of the SHG signal to be detected. ${ }^{34}$

The SHG signal was generated by the output of a modelocked Ti-sapphire laser with a repetition frequency of 82 $\mathrm{MHz}$, a pulse width of about $100 \mathrm{fs}$ and an average power on the sample between 100 and $250 \mathrm{~mW}$. The laser beam was focused onto the sample in a spot with diameter of 100 $-200 \mu \mathrm{m}$. The fundamental wavelength could be changed in the range of $0.71-0.84 \mu \mathrm{m}(\hbar \omega=1.48-1.75 \mathrm{eV})$. In this range, the linear optical absorption of the magnetic garnet films is in the order of $100 \mathrm{~cm}^{-1}$. Thus in transmission experiments the fundamental beam propagates through thin films without noticeable attenuation. On the other hand the linear absorption at the second harmonic energy around $3 \mathrm{eV}$ is very high, ${ }^{24,25}$ in the order of $10^{4}-10^{5} \mathrm{~cm}^{-1}$. Therefore in all samples, independently on their thickness, the detected SHG signal originates only from a back-side layer with a thickness less than $1 \mu \mathrm{m}$. Under such circumstances phasematching conditions are unimportant. ${ }^{3}$

The polarization of the incident fundamental light was first rectified with a Glan-laser polarizer then set with the help of a Babinet-Soleil compensator to the required linear or circular polarization. The error in the polarization due to the finite bandwidth of femtosecond pulses was estimated to be smaller than $10^{-2}$.

\section{EXPERIMENTAL RESULTS AND DISCUSSION}

\section{A. Effects of crystal symmetry due to the nonlinear tensor $\hat{\chi}^{\text {cr }}$}

According to the symmetry considerations given in Sec. II, SHG at normal incidence can be expected only in (111) and (210) oriented films, while those grown on (001) and (110) substrates should not show any SHG in a demagnetized state. We found that the SHG signals from the (111) and (210) films were in excellent agreement with the theory. In the (001) film, no SHG signal was detected at normal incidence. Contrary to expectation, the (110) films showed SHG signals in all measured samples. This is direct evidence of the fact that (001), (111), and (210) films grow epitaxially and reproduce the symmetry of the substrate surface, whereas this appears not to be the case for the (110) films.

Experimental results on SHG rotational anisotropy are given below for garnet films grown on substrates having different crystallographic orientations. The SHG rotational anisotropy pattern can be described by Eqs. (11). The symmetry of this pattern should by equal or higher than the corresponding crystallographic symmetry due to the Neumann principle.

(001) film (PG 4mm). Films with this orientation showed no crystallographic contribution to the SHG at all, in agreement with the symmetry predictions (see Table II).

(111) film (PG 3m). According to Table II, Eqs. (11) become

$$
\begin{gathered}
P_{X X}^{c r}=E_{X}^{2} A \cos 3 \varphi, \quad P_{X Y}^{c r}=E_{X}^{2} A \sin 3 \varphi, \\
P_{Y X}^{c r}=-E_{Y}^{2} A \cos 3 \varphi, \quad P_{Y Y}^{c r}=-E_{Y}^{2} A \sin 3 \varphi .
\end{gathered}
$$



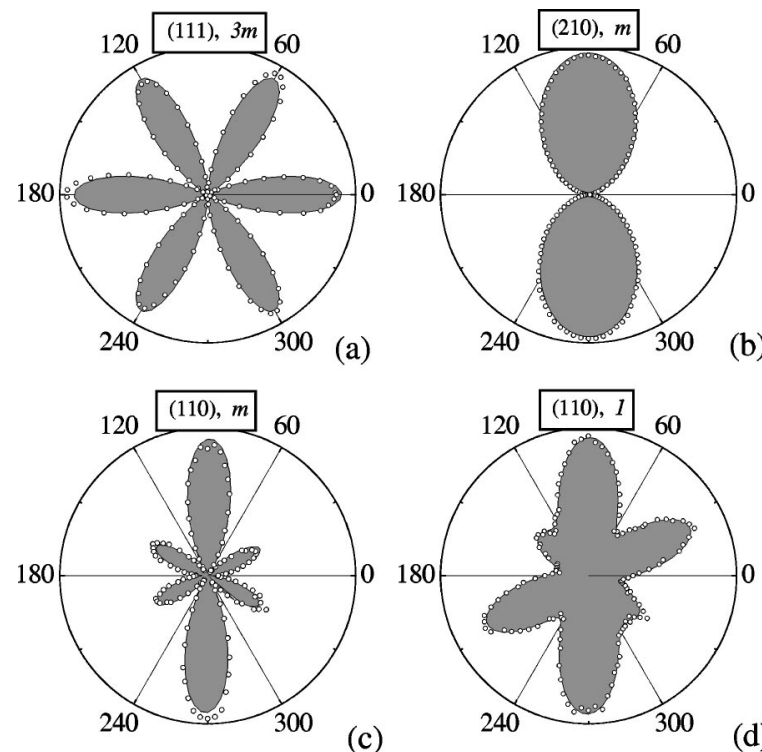

(d)

FIG. 3. SHG in garnet films with different crystallographic symmetry for the $X X$ polarization combinations: nonmagnetic case. The substrate orientation and the point group symmetry are indicated in the figure.

For the SHG intensity we get $I(2 \omega) \propto \cos ^{2} 3 \varphi$ for $X X$ and $Y X$ input-output polarization combinations and $I(2 \omega) \propto \sin ^{2} 3 \varphi$ for $X Y$ and $Y Y$ polarization combinations. Thus, a sixfold symmetry pattern should indicate the correct $3 \mathrm{~m}$ symmetry. This is indeed observed experimentally [see Fig. 3(a)]. In Fig. 3 experimental data are shown by circles and calculated values based on Eq. $I(2 \omega) \propto\left|P_{I J}^{c r}\right|^{2}$ are shown by lines.

The symmetry of the SHG rotational anisotropy pattern is higher than that of the original crystal (sixfold compared to threefold). This occurs because the SHG intensity is measured instead of the nonlinear polarization. Information about the phase of SHG can be obtained with the help of an external Ref. 35.

Another important point is the angular dependance of SHG polarization. From Eqs. (19) the angle of the polarization plane of the SHG light can be written as $\psi$ $=\arctan \left(P_{X Y}^{c r} / P_{X X}^{c r}\right)=3 \varphi$ for $X$-polarized excitation and as $\psi$ $=\arctan \left(P_{Y X}^{c r} / P_{Y Y}^{c r}\right)=\pi-3 \varphi$ for $Y$-polarized fundamental light. This means that the rotation of the sample by an angle $\varphi$ leads to the rotation of the SHG polarization plane by an angle $3 \varphi$.

(210) film (PG $m$ ). For this symmetry the nonlinear polarization of the medium is

$$
\begin{gathered}
P_{X X}^{c r}=E_{X}^{2}\left[(2 C-B+D) \sin ^{3} \varphi-(D+2 C) \sin \varphi\right], \\
P_{X Y}^{c r}=E_{X}^{2}\left[(2 C-B+D) \cos ^{3} \varphi+(B-2 C) \cos \varphi\right], \\
P_{Y X}^{c r}=E_{Y}^{2}\left[-(2 C-B+D) \sin ^{3} \varphi-(B-2 C) \sin \varphi\right], \\
P_{Y Y}^{c r}=E_{Y}^{2}\left[-(2 C-B+D) \cos ^{3} \varphi+(D+2 C) \cos \varphi\right] .
\end{gathered}
$$

These equations describe very well the experimental data on the SHG rotational anisotropy for films of this symmetry with three complex fitting parameters [see Fig. 3(b)].

(110) film (PG $m m 2, m$, or 1?). For this particular orientation of the film one should not expect any SHG signal at normal incidence based on the expected $\mathrm{mm} 2$ symmetry. However, this is not confirmed by our experiments. This means that the actual symmetry of these films is lower than $m m 2$. The SHG rotational anisotropy pattern by itself should be able to reveal the real symmetry of the film. The anisotropy patterns for two different (110) films (see Table III) are shown in Figs. 3(c) and 3(d). Clearly, in both cases there is a pronounced SHG signal comparable in magnitude to that observed in the (111) film. While the first one (No. 5) can be described by the formulas for the symmetry group $m$, data for the second film (No. 6) are in agreement with a complete absence of any symmetry, i.e., the crystallographic PG is 1 .

To summarize here, the experimental data on the SHG rotational anisotropy are in most cases in agreement with the expected crystallographic PG's. Strong deviations are, however, observed for (110) films, which must be due to structural distortions of the films for this particular orientation. One may conclude that the SHG pattern by itself yields the real PG symmetry of the given samples which is very difficult or even impossible to get from $\mathrm{x}$-ray data.

\section{B. Influence of magnetization on SHG in the transversal geometry}

The presence of a spontaneous or magnetic-field induced magnetization in a sample leads to the breaking of timereversal symmetry and gives rise to the presence of a nonlinear optical tensor $\hat{\chi}^{m}$. Now, interference between the nonlinear optical waves of crystallographic and magnetic origin becomes a very essential part of the SHG process. Equation (18) describes the total SHG intensity. The effects from the crystallographic part have been discussed above, as for the magnetic effects, we consider first the transversal geometry, where the linear magneto-optical effects do not contribute (see Sec. II).

\section{Nonlinear magneto-optical effects linear in $\mathrm{M}$}

The experimental results show that there is a nonlinear magneto-optical effect which is linear in magnetization..$^{30,31}$ Such a novel nonlinear magneto-optical effect is a direct consequence of the interference between optical waves having different nonlinear source polarizations $\mathbf{P}^{c r}$ and $\mathbf{P}^{m}$. Note, in the linear case, in the transversal geometry at normal incidence only effects that are proportional to $M^{2}$, such as magnetic linear birefringence, can be observed.

Figure 4 shows the rotational anisotropy patterns for the (210) film that supports the conclusion about the crystallographic PG $m$. For this symmetry, Eqs. (15) give nonzero magnetic contributions for all four polarization combinations of incoming-outgoing light. Indeed, in our experiments very strong changes of SHG intensity have been observed due to the magnetization reversal.

Comparing the plots of Fig. 4 with Fig. 3(b) one clearly observes the disappearance of one symmetry plane of the 

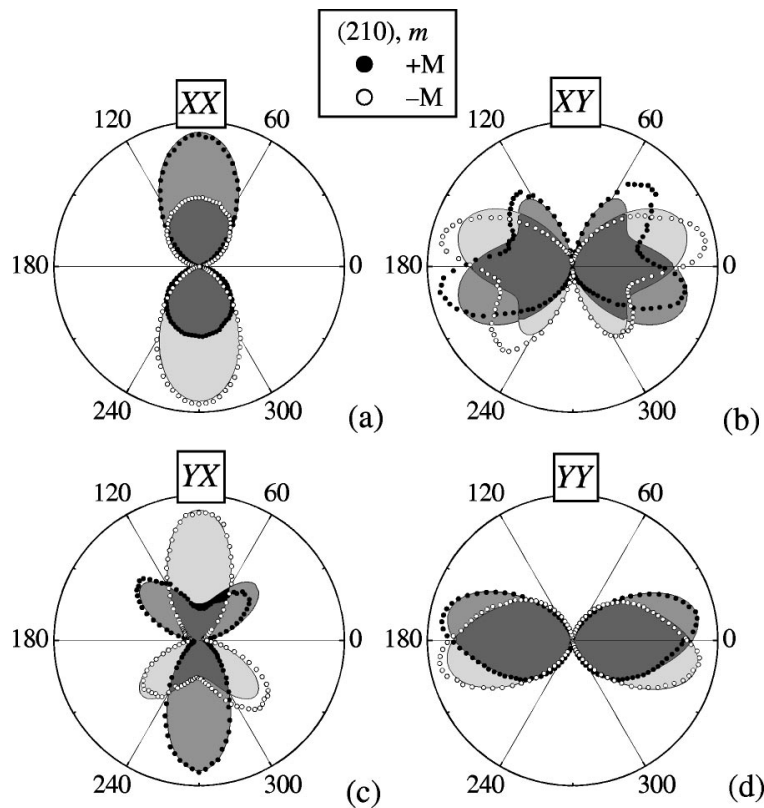

FIG. 4. Transversal nonlinear magneto-optical effect in a (210)oriented film for the four polarization combinations. The experimental data are shown by circles and the best fit based on Eqs. (12), (16), and (19) is shown by lines. The light gray shadowed area is shown for $-\mathbf{M}$, the gray shadowed area is shown for $+\mathbf{M}$ and overlapped area is shown by the dark gray.

rotational anisotropy pattern: each of the plots has symmetry $m$. However, in addition to that we can also find the symmetry plane $m^{\prime}$, i.e., reflection plane symmetry in combination with the time-reversal operation. These two types of planes are mutually perpendicular to one another.

(110) films must be discussed separately. In Sec. IV A we have shown, based on that the crystallographic contribution to the SHG, that these films have PG symmetry either $m$ or 1 The experimental data for the magnetized (110) film (see Fig. 5) also show a presence of SHG interference effects. This interference makes it possible to distinguish two magnetization states $+\mathbf{M}$ and $-\mathbf{M}$. We found that the SHG rotational anisotropy for the (110) films can be well described by the same equations for the crystal PG symmetry $m$ or 1 though the rotational patterns of these types of films look differently (Fig. 5). This proves the earlier conclusion about the symmetry lowering for the (110) films. In Fig. 5 the experimental data are shown by solid and open circles for the two magnetization states $+\mathbf{M}$ and $-\mathbf{M}$, respectively, and the best fit is shown by the solid line for $+\mathbf{M}$ and the dashed line for $-\mathbf{M}$.

\section{Nonlinear optical effects proportional to $M^{2}$}

For (001) films the PG is $4 \mathrm{~mm}$ leading to the absence of a nonlinear crystallographic contribution at normal incidence. This means that for this film one can expect only a magnetization-induced SHG, and the SHG intensity should not be sensitive to the magnetization reversal.

As mentioned above, in the (001) film no SHG signal was detected in the absence of a magnetic field. In this case the film has a magnetic domain structure with the magnetization
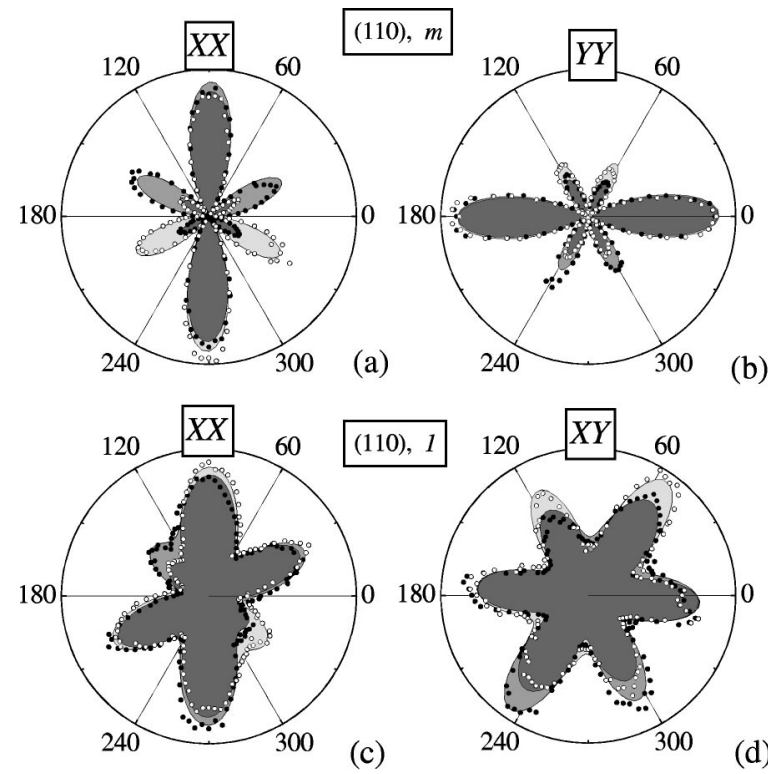

FIG. 5. Transversal nonlinear magneto-optical effect in (110)oriented films (a), (b) symmetry $m$, (c), (d) symmetry 1 .

orientation along the $z$ axis, therefore there is no magnetic contribution to the SHG (see Table II). However, as predicted, in a magnetized sample $(\mathbf{M} \| Y)$ an SHG signal was observed, which was quadratic in the magnetization $\mathbf{M}$ and thus insensitive to the sign of the applied magnetic field. ${ }^{30,31}$ Thus, the SHG response can be "switched on" with the help of an external transversal magnetic field.

\section{Nonlinear magneto-optical effects in the longitudinal geometry}

In the longitudinal geometry the Faraday effect results in the rotation of the polarization of the light at the fundamental and SHG frequencies. Although the Faraday rotation is quite strong at the double frequency, ${ }^{24}$ the very small thickness of the layer (less than $1 \mu \mathrm{m}$ ) from which the SHG is emitted, makes the rotation at $2 \omega$ considerably smaller than that at the fundamental frequency. Therefore, one can take into account the polarization rotation at the fundamental frequency (which is easy to measure) and neglect the same effect at the double frequency. For the calculation of SHG intensity Eq. (18) can be used taking into account the Faraday rotation at the fundamental frequency. The interference of crystallographic [Eqs. (11)] and magnetic [Eqs. (16)] parts leads to effects linear in $\mathbf{M}$.

\section{Nonlinear optical rotation and ellipticity linear in $\mathbf{M}$}

In the longitudinal geometry the SHG intensity in the (111) film (PG 3m) can be written as

$$
\begin{gathered}
I_{X X}(2 \omega) \propto E_{X}^{4}\left|A \cos 3 \varphi+m M_{Z} \sin 3 \varphi\right|^{2}, \\
I_{X Y}(2 \omega) \propto E_{X}^{4}\left|A \sin 3 \varphi-m M_{Z} \cos 3 \varphi\right|^{2}, \\
I_{Y X}(2 \omega) \propto E_{Y}^{4}\left|-A \cos 3 \varphi-m M_{Z} \sin 3 \varphi\right|^{2},
\end{gathered}
$$




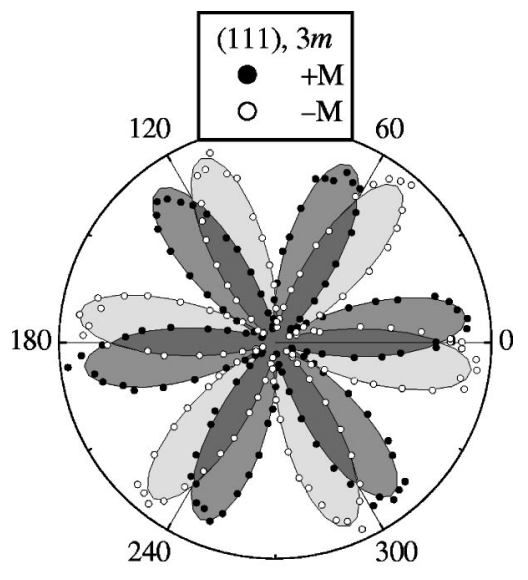

FIG. 6. SHG rotational anisotropy pattern in a (111)-oriented film in the longitudinal configuration.

$$
I_{Y Y}(2 \omega) \propto E_{Y}^{4}\left|-A \sin 3 \varphi+m M_{Z} \cos 3 \varphi\right|^{2} .
$$

In the transversal geometry the magnetic contribution is isotropic for $X X$ polarizations and equal to zero for $Y Y$ polarizations in this film. In the longitudinal geometry this is not the case any more. The interference between crystallographic and magnetic contributions to the SHG leads to the rotation of the sixfold pattern as a whole for all polarization combinations. From the experimentally observed rotation (see Fig. 6 ), having information about the linear Faraday rotation at the fundamental frequency, one could estimate the nonlinear rotation value of SHG light polarization.

Thus, in a certain sense the nonlinear rotation effect is similar to the linear Faraday effect. The value of the nonlinear rotation angle can be written as

$$
\alpha=-\arctan \left(\operatorname{Re} \frac{m M_{Z}}{A}\right) .
$$

The value of the nonlinear ellipticity is

$$
\epsilon=-\arctan \left(\operatorname{Im} \frac{m M_{Z}}{A}\right) .
$$

For $m M_{Z} \ll A$ the nonlinear rotation $\alpha$ and ellipticity $\epsilon$ are linear functions of the magnetization $\mathbf{M}$.

In Fig. 7 the SHG intensity is shown for four polarization combinations in the (210) film (PG $m$ ). Effects of the nonlinear rotation and ellipticity in this film are quite different to those observed in the (111) films due to the lower symmetry. The experimental results where fitted with Eqs. (11), (16), and (18) using relevant components of the nonlinear optical tensors from Tables I and II. In Figs. 6 and 7 experimental data are shown by circles, the best fit is shown by lines.

\section{Nonlinear magneto-optical asymmetry under circular excitation}

In the limit of a thin magnetic film and therefore neglecting the magnetic circular dichroism at $2 \omega$, using Eqs. (13) and (17) the SHG intensity for circular excitation can be written as
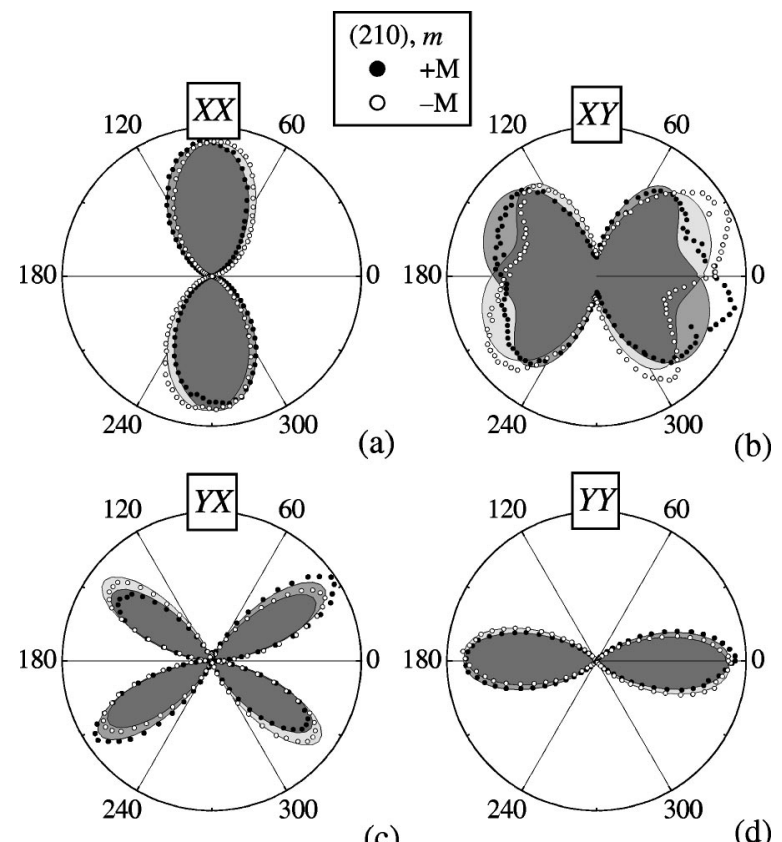

(d)

FIG. 7. SHG rotational anisotropy patterns in the longitudinal geometry for (210)-oriented films for the four polarization combinations.

$$
\begin{aligned}
I_{C}(2 \omega) \propto & E_{0}^{4}\left[\left|A-G \pm 2 i C+(l-t) M_{z} \pm 2 i n M_{z}\right|^{2}\right. \\
& \left.+\left|D-B \pm 2 i F+(p-m) M_{z} \pm 2 i s M_{z}\right|^{2}\right],
\end{aligned}
$$

where the \pm sign refers to left/right circular polarization of the fundamental light, respectively. Therefore, the SHG intensity depends on the helicity of the fundamental light. Figure 8 summarize observed nonlinear optical effects. In the films having symmetry $3 m$ circularly polarized incident light produces circularly polarized SHG with reversible nonlinear asymmetry in respect to the light helicity and magnetization direction change. Lowering the symmetry leads to elliptical polarization of the outgoing SHG light [see Fig. 8(a) for the (210) film] and even to the nonlinear asymmetry with no equivalence between light helicity and magnetization direction change for the (110) film [Fig. 8(b)]. In more details,

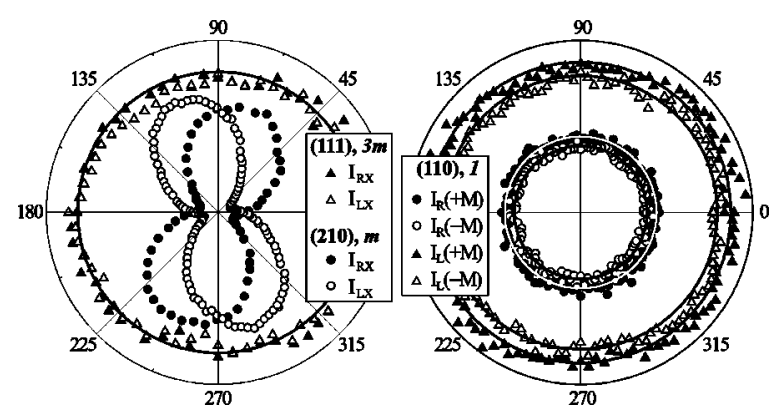

FIG. 8. SHG rotational anisotropy patterns measured with circularly polarized fundamental light in films of three different symmetries. 
results on SHG for garnet films of (111), (210), and (110) orientation in the longitudinal geometry are presented in Ref. 36.

Clearly, the symmetry approach gives a possibility to qualitatively account for all arising nonlinear magnetooptical effects. However, for a more quantitative description in the longitudinal geometry, the problem of nonlinear propagation of light in magnetized nonlinear media discussed in Sec. II B should be solved. A computer program was used to simulate the SHG intensity because an explicit analytical expression could not be derived. As the input parameters to the program, the optical and magneto-optical parameters of the samples should be given for both $\omega$ and $2 \omega$ frequencies. While for the fundamental wavelength all parameters are easy to measure for the given samples, this is not the case for the SHG frequency because of the very strong absorption. Therefore, in this case it is difficult to give precise quantitative data. It appears that taking into account the complete propagation-generation effects leads to the rescaling of the purely SHG contribution into the total effects. However on the qualitative level, no difference was found from the simple symmetry approach.

\section{CONCLUSION}

The present work gives a theoretical analysis and experimental observation of the nonlinear magneto-optical phenomena related to the process of SHG in anisotropic magnetic films. In noncentrosymmetric crystals in the electricdipole approximation two kinds of optical nonlinearity coexist. The first is due to the charge ordering defined by the crystal structure whereas the second one is due to magnetic ordering. These two kinds of ordering may be regarded as independent sources of nonlinear optical waves. The interference between nonlinear waves gives rise to new magnetooptical phenomena, which have no counterparts in linear optics. The theoretical model given for nonlinear propagation of light can be applied for longitudinal and transversal geometries for an external magnetic field.

Epitaxial films of magnetic garnets grown on substrates with (100), (110), (111), and (210) crystallographic orientations were taken as a model system for experimental studies of the novel nonlinear magneto-optical effects. Using a mode-locked Ti:sapphire laser tunable in the range 1.48 $-1.75 \mathrm{eV}$ we were able to detect SHG signals in the trans- mission geometry with high signal-to-noise ratio. We show that crystallographic and magnetic contributions to the SHG can be unambiguously separated with the help of rotational anisotropy experiments in an applied magnetic field. The rotational anisotropy pattern of SHG allows the determination of the epitaxial film symmetry. The crystal symmetry was found to be $4 \mathrm{~mm}$ for (100) films, $3 \mathrm{~m}$ for (111) films, and $m$ for (210) films. In all these cases the film symmetry is a product of the corresponding crystal surface symmetry and the symmetry of a polar vector perpendicular to the crystal surface. In contrast, in (110) films the symmetry was lower than expected. Instead of the expected $\mathrm{mm} 2$ symmetry the anisotropy pattern showed $m$ or even 1 symmetry groups. This might happen due to structural distortions of the (110)oriented films.

In this paper we explored mainly the macroscopical properties of SHG in epitaxial magnetic films and could explain them assuming two sources of nonlinear optical polarization related to the crystallographic and magnetic contributions and interference between them. Surprisingly, these two contributions were found to be of the same order of magnitude. The largest SHG signals were observed in Bi-containing (210) films characterized by a larger misfit between the substrate and the film. Microscopic mechanisms of crystallographic and magnetic sources of SHG remained out of the scope of this paper and obviously should become a subject of future studies. Important problems to solve are the mechanisms of the space-inversion symmetry breaking and the symmetry lowering in epitaxial films possessing a crystallographic structure which originates from the cubic centrosymmetric garnet structure. Possible structural mechanisms were recently analyzed in Refs. 37,38, where it was shown that the lattice misfit strain and misfit dislocation strain may contribute to the second order nonlinear optical susceptibility due to a nonlinear photoelastic effect.

\section{ACKNOWLEDGMENTS}

Part of this work was supported by HCM Grants No. ERBCHBGCT930444 and ERBCHRXCT940563, INTAS 970705, the European TMR network ERBFMRXCT98-0015 (NOMOKE), the Dutch-Russian Project NWO, and the Russian Foundation for Basic Research. V.V.P. and R.V.P. acknowledge the financial support of RIM during their stay at the University of Nijmegen.
*Email address: kirilyuk@sci.kun.nl

${ }^{1}$ P. A. Franken, A. E. Hill, C. W. Peters, and G. Weinreich, Phys. Rev. Lett. 7, 118 (1961).

${ }^{2}$ N. Bloembergen, Nonlinear Optics (Benjamin, New York, 1965).

${ }^{3}$ Y. R. Shen, The Principles of Nonlinear Optics (Wiley, New York, 1984).

${ }^{4}$ R. W. Boyd, Nonlinear Optics (Academic Press, London, 1992).

${ }^{5}$ Nonlinear Optics in Metals, edited by K. H. Bennemann (Clarendon Press, Oxford, 1998).

${ }^{6}$ T. F. Heinz, in Nonlinear Surface Electromagnetic Phenomena, edited by H.-E. Ponath and G. I. Stegeman (North Holland, Amsterdam, 1991).
${ }^{7}$ G. A. Reider and T. F. Heinz, in Photonic Probes of Surfaces, edited by P. Halevi (Elsevier, Amsterdam, 1995).

${ }^{8}$ A. K. Zvezdin and V. A. Kotov, Modern Magnetooptics and Magnetooptical Materials (IOP Publishing, Bristol, 1997).

${ }^{9}$ P. S. Pershan, Phys. Rev. 130, 919 (1963).

${ }^{10}$ E. Adler, Phys. Rev. 134, A728 (1964).

${ }^{11}$ S. Kielich and R. Zawodny, Acta Phys. Pol. A 43, 579 (1973); Opt. Acta 20, 867 (1973).

${ }^{12}$ N. N. Akhmediev, S. B. Borisov, A. K. Zvezdin, I. L. Lyubchanskii, and Yu. V. Melikhov, Sov. Phys. Solid State 27, 650 (1985).

${ }^{13}$ Ru-Pin Pan, H. D. Wei, and Y. R. Shen, Phys. Rev. B 39, 1229 
(1989).

${ }^{14}$ W. Hübner and K. H. Bennemann, Phys. Rev. B 40, 5973 (1989).

${ }^{15}$ J. Reif, J. C. Zink, C. M. Schneider, and J. Kirschner, Phys. Rev. Lett. 67, 2878 (1991).

${ }^{16}$ J. Reif, C. Rau, and E. Matthias, Phys. Rev. Lett. 71, 1931 (1993).

${ }^{17}$ G. Spierings, V. Koutsos, H. A. Wierenga, M. W. J. Prins, D. Abraham, and Th. Rasing, Surf. Sci. 287/288, 747 (1993); J. Magn. Magn. Mater. 121, 109 (1993).

${ }^{18}$ H. A. Wierenga, W. de Jong, M. W. J. Prins, Th. Rasing, R. Vollmer, A. Kirilyuk, H. Schwabe, and J. Kirschner, Phys. Rev. Lett. 74, 1462 (1995).

${ }^{19}$ M. Straub, R. Vollmer, and J. Kirschner, Phys. Rev. Lett. 77, 743 (1996).

${ }^{20}$ B. Koopmans, M. Groot Koerkamp, Th. Rasing, and H. van den Berg, Phys. Rev. Lett. 74, 3692 (1995).

${ }^{21}$ M. Fiebig, D. Fröhlich, B. B. Krichevtsov, and R. V. Pisarev, Phys. Rev. Lett. 73, 2127 (1994).

${ }^{22}$ M. Fiebig, D. Fröhlich, G. Sluyterman v. L., and R. V. Pisarev, Appl. Phys. Lett. 22, 2906 (1995).

${ }^{23}$ Physics of Magnetic Garnets, edited by A. Paoletti (North Holland, Amsterdam, 1978).

${ }^{24}$ G. Winkler, Magnetic Garnets (Vieweg, Braunschweig, 1981).

${ }^{25}$ Magnetic Garnet Films, guest editor A. Paoletti, special issue of Thin Solid Films 114 (1984).

${ }^{26}$ Landolt-Börnstein, Numerical Data and Functional Relationships in Science and Technology, New Series, Group III, 27/e (Springer-Verlag, Berlin, 1991).
${ }^{27}$ O. A. Aktsipetrov, O. V. Braginskii, and D. A. Esikov, Sov. J. Quantum Electron. 20, 259 (1990).

${ }^{28}$ R. V. Pisarev, B. B. Krichevtsov, V. N. Gridnev, V. P. Klin, D. Fröhlich, and Ch. Pahlke-Lerche, J. Phys. C 5, 8621 (1993).

${ }^{29}$ G. Petrocelli, S. Martelucci, and M. Richetta, Appl. Phys. Lett. 63, 3402 (1993).

${ }^{30}$ R. V. Pisarev, V. V. Pavlov, A. Kirilyuk, and Th. Rasing, J. Magn. Soc. Jpn. 20, 23 (1996).

${ }^{31}$ V. V. Pavlov, R. V. Pisarev, A. Kirilyuk, and Th. Rasing, Phys. Rev. Lett. 78, 2004 (1997).

${ }^{32}$ V. N. Gridnev, Solid State Commun. 100, 71 (1996).

${ }^{33}$ B. B. Krichevtsov, V. V. Pavlov, and R. V. Pisarev, JETP Lett. 49, 535 (1989); Sov. Phys. Solid State 31, 1142 (1989).

${ }^{34}$ This method, however, left the magnetic contribution that is proportional to $M^{2}$ unresolved. In order to really select the crystallographic contribution alone, SHG signals were measured as a function of the sample temperature (Ref. 31). Above $T_{C}$ all magnetic contributions disappeared.

${ }^{35}$ K. J. Veenstra, A. V. Petukhov, A. P. de Boer, and Th. Rasing, Phys. Rev. B 58, R16 020 (1998).

${ }^{36}$ A. Kirilyuk, V. V. Pavlov, R. V. Pisarev, and Th. Rasing, Phys. Rev. B 61, R3796 (2000).

${ }^{37}$ I. L. Lyubchanskii, N. N. Dadoenkova, M. I. Lyubchanskii, Th. Rasing, Jae-Woo Jeong, and Sung-Chul Shin, Appl. Phys. Lett. 76, 1848 (2000).

${ }^{38}$ I. L. Lyubchanskii, Jae-Woo Jeong, Sung-Chul Shin, N. N. Dadoenkova, M. I. Lyubchanskii, and Th. Rasing, J. Appl. Phys. 87, 6794 (2000). 